\title{
Excitation and dissipation of torsional modes in solar photospheric magnetic flux tubes
}

\author{
Q. Y. Luo, F. S. Wei, and X. S. Feng \\ Key Laboratory for Space Weather, CAS, POB 8701, Beijing 100080, PR China \\ Received 26 March 2002 /Accepted 4 July 2002

\begin{abstract}
The turbulent excitation of torsional Alfvén waves is presented on the basis of Lighthill-Stein theory. Due to the special properties of torsional modes, we can apply the theory to the inhomogeneous magnetic flux tube embedded in the solar photosphere, to evaluate the wave energy generated from the turbulent source in the convection zone. Torsional wave spectrum distribution along the $r$ coordinate is achieved. In order to estimate the net wave energy transported to the chromosphere, we investigate the wave propagation and dissipation in the photospheric flux tube by simple phase mixing due to the inhomogeneity. Results for several cases with different model parameters are compared. Conclusions are drawn on the significant role of the energy carried by torsional Alfvén waves in the chromospheric and coronal heating, and the wave spectrum may be helpful in understanding the problem of footpoint azimuthal motion of coronal loops.
\end{abstract}

Key words. waves - Sun: photosphere - Sun: magnetic fields

\section{Introduction}

Magnetic flux tubes are ubiquitous in space and in solar plasma, from small-scale structures like supergranulation, networks, coronal loops, and sunspots, to large-scale structures like prominences and coronal holes, all of which can be analyzed as tube topology. With more observations available from spacecrafts such as SOHO, TRACE, and ground stations, we get more data to understand the importance of magnetic flux tubes in the activities of the sun, e.g., explosive and transient phenomena. Hence, magnetic flux tubes are an essential subject in current space and solar physics. The dynamic motion of magnetic flux tubes was investigated in the convection zone and chromosphere in the classic paper by Spruit (1981), and the tube wave modes were discussed in Spruit (1982). More recently, Roberts \& Ulmschneider (1997) reviewed extensively the research on the various dynamical processes and wave effects in magnetic flux tubes.

The mechanism of coronal heating has been an open question for many years, and the particular effects of magnetohydrodynamic (MHD) waves in the magnetic flux tubes are believed to play roles here. Many efforts have been made (De Groof et al. 2002; Sakai et al. 2000; Berghmans \& De Bruyne 1995; Berghmans \& Tirry 1997). The original work on the wave generation from turbulence, which concerns us most, was by Lighthill (1952), in which he studied the excitation of acoustic waves from fluid turbulence. Stein (1967) developed this wave excitation theory, and applied it to MHD wave generation. Furthermore, Collins (1989a,b, 1992) explored the general property of flux tube wave modes in the case of the

Send offprint requests to: $\mathrm{Q}$. Y. Luo,

e-mail: qyluo@ns.lhp.ac.cn period velocity field. Lighthill-Stein theory was also extended by Musielak et al. (1989), and his results highlighted the enhancement of MHD wave generation in magnetic flux tubes. More applications of the turbulent excitation theory had been applied to research on sunspots (Lee 1993), late-type dwarfs and subgiants (Musielak et al. 2000).

In the AC heating mechanism of chromosphere and corona, Alfvén waves are the most hopeful candidates to carry energy from the convection zone to the upper atmosphere. Therefore, Alfvén waves are the key element in the problem of MHD wave propagation in the solar atmosphere (Hollweg 1991). The dissipation mechanism of these waves have also been studied with many theories such as resonance absorption, shock dissipation, turbulent dissipation, nonlinear evolution, etc. After being first proposed by Heyvaerts \& Priest (1983), phase mixing was accepted as one of the most important dissipation mechanisms of Alfvén waves, and was applied to explain the heating of coronal and solar winds. Ruderman et al. (1999) investigated the phase mixing effect in the two dimensionally axisymmetric magnetic flux tube with steady flow, and provided a possible description of the wave energy supply. More research is reviewed in Narain \& Ulmschneider (1990, 1996).

In this paper, we first explore the problem of incompressible linear torsional Alfvén waves generated in the inhomogeneous magnetic flux tube, where the wave source is the turbulent motion located in the convection zone. To tackle the problem, we describe the whole flux tube as being divided into a bunch of "elementary tubes" (see Sect. 2), where the plasma is uniform. In the process of wave excitation, the interference among the waves in different elementary tubes can be ignored under the assumption of a strong turbulent source model and the $\theta$ independence of the torsional mode. Then we 
can obtain the torsional wave energy flux in the elementary tube using Lighthill-Stein theory, and the waves are generated from the local turbulent source by the resonance excitation (unlike the work of Musielak, who studied the longitudinal and transverse modes). Due to the lack of knowledge about turbulence features in the convection zone, similar to previous work, the Kolmogorov turbulent spectrum is taken as the description of our source model. To estimate the net wave energy flux that can transfer through the photosphere, we evaluate the wave energy dissipation by taking account of the simple phase mixing effect. Finally, the magnitude of the torsional wave energy, which is available in the heating of chromosphere and corona, is given in our results.

In the next section, we give a brief introduction to the wave modes in magnetic flux tubes, and describe the inhomogeneous tube model in detail. In Sect. 3, we analyze the torsional Alfvén wave energy flux in the local elementary flux tube. Then in Sect. 4, to estimate the dissipation of the torsional waves, we employ the Heyvaerts and Priest result about phase mixing. Section 5 presents our results and relevant discussion and Sect. 6 our conclusions.

\section{Torsional modes in magnetic flux tubes}

The basic modes of oscillation in magnetic flux tubes are determined by the property of the tube magnetic configuration. In terms of the geometrical topology, we distinguish them into sausage modes, kink modes, and fluting modes; in terms of the fluctuation pattern, they may be longitudinal modes, transverse modes, and torsional modes. For more details, see Roberts \& Ulmschneider (1997).

Based on the Lighthill-Stein theory, the MHD wave energy flux generated from turbulent motion was studied for different modes: longitudinal modes in Musielak et al. (1989, 1995), transverse modes in Musielak \& Ulmschneider (2001). Here, what we are concerned with is the dynamical process of excitation and dissipation of the linear incompressible torsional Alfvén mode in flux tubes.

We consider an individual untwisted magnetic flux tube, vertically oriented, with a circular section and initial internal axial magnetic field $\boldsymbol{B}_{0}\left(=B_{0}(r) e_{z}\right)$, and in equilibrium with the surrounding medium. The magnetic field $\boldsymbol{B}_{0}(r)$ in the tube declines with radial coordinate $r$ according to

$B_{0}(r)=B_{0} \mathrm{e}^{-\alpha r / R_{0}}$,

where $R_{0}$ is the radius of the tube, $B_{0}$ the initial magnetic field at tube center, and $\alpha$ the radial declining coefficient of the magnetic field. It is remarkable that the radial declining coefficient $\alpha$ is a description of the magnetic inhomogeneity, as well as a description of the tube edge definition. The equilibrium condition can be easily expressed as a horizontal pressure balance,

$\frac{B_{0}^{2}(r)}{2 \mu_{0}}+p_{0}(r)=p_{\mathrm{e}}$,

where $p_{0}$ and $p_{\mathrm{e}}$ are the fluid pressures inside and outside the flux tube, respectively.

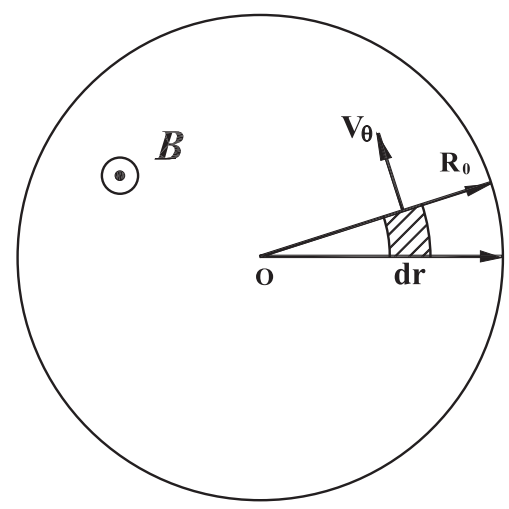

Fig. 1. The elementary tube, as showed with the shaded area. $R_{0}$ is the radius of the inhomogeneous magnetic flux tube, $B$ the magnetic field declining with increasing radial coordinate, and $V_{\theta}$ the velocity perturbation.

In our problem, we neglect the solar atmosphere stratification due to gravitational force, and concentrate on the dynamical process of torsional modes, which is not so realistic but simplifies the problem considerably.

During the derivation of wave excitation, we assume that the resistivity and viscosity of the magnetofluid can be ignored on small spatial scales. Also, similar to the procedure of Nakariakov et al. (1997) but in cylindrical coordinates, the MHD equations of the $\theta$ component for the inhomogeneous flux tube are obtained,

$\rho_{0} \frac{\partial}{\partial t} v_{\theta}-\frac{1}{\mu_{0}} B_{0}(r) \frac{\partial}{\partial z} B_{\theta}=-\rho_{0} v_{z} \frac{\partial}{\partial z} v_{\theta}+\frac{1}{\mu_{0}} B_{z} \frac{\partial}{\partial z} B_{\theta}$,

$\frac{\partial}{\partial t} B_{\theta}-B_{0}(r) \frac{\partial}{\partial z} v_{\theta}=\frac{\partial}{\partial z}\left(B_{z} v_{\theta}-v_{z} B_{\theta}\right)$.

In the above expressions, $\rho_{0}$ is the hydromagnetic density, $B_{0}(r)$ the initial local magnetic field, and the variables with subscript $\theta, z$ represent the field perturbations in their respective directions. The nonlinear terms on the right hand sides are treated subsequently as turbulent sources. In deriving the set of equations above, we have made use of the axisymmetry assumption, $\partial / \partial \theta=0$; and to simplify the problem, the $\theta$ component is supposed to be dominant in the transverse turbulent source terms.

Next, we will employ the MHD Eqs. (3), (4) in the local dynamical wave excitation. As showed in Fig. 1, an elementary portion can be separated from the inhomogeneous magnetic flux tube, and the variation of field parameters in this zone can be neglected. Here we decouple the torsional Alfvén wave from the magnetosonic waves by the constraint $k_{\mathrm{r}}=0$. And obviously, if we apply Eqs. (3), (4) to the local elementary portion, shown by the shaded area in Fig. 1, the $\theta$ component of perturbation can be replaced by the transverse $x$ component in the "elementary tube", as defined in this paper. In other words, the torsional modes in the whole magnetic flux tube can be considered as the sum of local waves generated from the local turbulence source. Furthermore, as the premise of the LighthillStein theory, the strong turbulence pressure is assumed to be overwhelming in the nonlinear terms (see Musielak et al. 1995), i.e., the right hand sides of Eqs. (3), (4). In this sense, we 
can omit the effect from the interference of the waves among different elementary tubes during the process of wave excitation. Therefore, with these descriptions above, we assume that the efficiency of wave excitation can only be directly determined by the local hydromagnetic parameters and the turbulence strength. In what follows, we shall derive the generation of torsional Alfvén waves in an individual elementary tube first.

\section{Local turbulent excitation of torsional Alfvén waves}

In this section, we deduce the energy flux of torsional Alfvén waves generated from the local turbulent source.

\subsection{Wave equation and wave energy flux}

From the discussion in Sect. 2, if the $\theta$ components of perturbations in Eqs. (3), (4) are replaced by the $x$ components in the elementary tube, we get the wave equation

$\frac{\partial^{2}}{\partial t^{2}} v_{x}-c_{\mathrm{A}}^{2} \frac{\partial^{2}}{\partial z^{2}} v_{x}=c_{\mathrm{A}}\left(\frac{\partial}{\partial z}\right)^{2}\left(u_{x} h_{z}-u_{z} h_{x}\right)-\frac{\partial^{2}}{\partial z \partial t}\left(u_{z} u_{x}-h_{x} h_{z}\right)$,

with local Alfvén velocity $c_{\mathrm{A}}$ defined as

$c_{\mathrm{A}}=B_{0}(r) / \sqrt{\mu_{0} \rho_{0}}$.

Here, $\boldsymbol{u}$ is the fluid turbulent velocity and $\boldsymbol{h}$ the turbulent Alfvén velocity, which is related to magnetic field perturbation $\boldsymbol{b}$ by

$\boldsymbol{h}=\boldsymbol{b} / \sqrt{\mu_{0} \rho_{0}}$.

During the derivation above, we assume that the turbulent field takes the primary role in the wave generation process, and in comparison, the interference effect on the generation of waves can be ignored as discussed earlier. We can consider mainly the turbulent excitation, and the Reynold stress (including the magnetic Reynold stress) is the unique source of wave generation. Thus, nonlinear terms on the right hand side of wave equation (Eq. (5)) can be treated as the turbulent terms, which is also the key point of the Lighthill-Stein theory.

The expression of wave energy flux in the elementary tube can be derived from the general definition of MHD energy flux,

$F(z, t)=-\frac{B_{0}}{\mu_{0}} B_{x}(z, t) v_{x}(z, t)$,

with

$B_{x}=\frac{\mu_{0} \rho_{0}}{B_{0}}\left(\frac{\partial}{\partial z}\right)^{-1}\left(\frac{\partial}{\partial t}\right) v_{x}$.

\subsection{Fourier transformation}

In the local elementary tube, we can Fourier-transform Eq. (5) into the $\left(k_{z}, \omega\right)$ space (hereafter, we explicitly drop the subscript $z$ in $k_{z}$ ),

$v_{x}(k, \omega)=\frac{S_{\mathrm{A}}(k, \omega)}{k^{2} c_{\mathrm{A}}^{2}-\omega^{2}}$, with the source term $S_{\mathrm{A}}$,

$$
\begin{aligned}
S_{\mathrm{A}}(k, \omega)= & \frac{1}{(2 \pi)^{2}} \iint \mathrm{d} z \mathrm{~d} t \\
& \times\left(k^{2} c_{\mathrm{A}}\left(u_{z} h_{x}-u_{x} h_{z}\right)+k \omega\left(u_{z} u_{x}-h_{z} h_{x}\right)\right) \mathrm{e}^{i(k z-\omega t)}
\end{aligned}
$$

After using inverse Fourier-transformations, we substitute Eqs. (9)-(11) into Eq. (8), and take the average with respect to time to obtain

$$
\begin{aligned}
<F(z, t)>_{t}= & -\rho_{0}<v_{x}^{*}\left(\frac{\partial}{\partial z}\right)^{-1}\left(\frac{\partial}{\partial t}\right) v_{z}>_{t} \\
= & \rho_{0} \lim _{t_{0} \rightarrow \infty} \frac{2 \pi}{t_{0}} \iint \mathrm{d} k^{\prime} \mathrm{d} k \int \mathrm{d} \omega \\
& \times \mathrm{e}^{-i\left(k-k^{\prime}\right)}\left(\frac{\omega}{k}\right) \frac{S_{\mathrm{A}}^{\prime}\left(k^{\prime}, \omega\right)}{k^{\prime 2} c_{\mathrm{A}}^{2}-\omega^{2}} \frac{S_{\mathrm{A}}(k, \omega)}{k^{2} c_{\mathrm{A}}^{2}-\omega^{2}} .
\end{aligned}
$$

Integrating the energy flux expression by the residual theorem, we get the following monochromatic wave energy flux,

$$
\frac{\partial}{\partial \omega} F(z, t)=<F(z, \omega)>=\frac{2 \pi \rho_{0}}{t_{0}} \frac{1}{c_{\mathrm{A}}^{4}} \frac{\pi^{2}}{4 k_{0}^{2}} \frac{\omega}{k_{0}}\left|S_{\mathrm{A}}\left(k_{0}, \omega\right)\right|^{2},
$$

where $k_{0}=\omega / c_{\mathrm{A}}$.

During the above process of resonance excitation, we neglected the wave propagating antiparallel to the $z$ direction. Then, we have the energy flux in Eq. (13), except for the expression of the source term, which is given in the next subsection.

\subsection{Source term}

Similar to Stein (1967), we take time and space average of the turbulent correlation terms, and substitute the arguments in Eq. (11) with

$r=z-z^{\prime}, \tau=t-t^{\prime}$

Then, we have the source term in Eq. (13),

$$
\begin{aligned}
& \left|S_{\mathrm{A}}\left(k_{0}, \omega\right)\right|^{2} \\
& =\frac{1}{(2 \pi)^{4}} \iiint \int \mathrm{d} z \mathrm{~d} z^{\prime} \mathrm{d} t \mathrm{~d} t^{\prime} \mathrm{e}^{i \omega\left(t-t^{\prime}\right)-i k_{0}\left(z-z^{\prime}\right)} \\
& \quad \times\left(k_{0}^{4} c_{\mathrm{A}}^{2}\left(<v_{x} v_{x}^{\prime} h_{z} h_{z}^{\prime}>+<v_{z} v_{z}^{\prime} h_{x} h_{x}^{\prime}>\right)\right. \\
& \left.\quad+k_{z}^{2} \omega^{2}\left(<v_{x} v_{x}^{\prime} v_{z} v_{z}^{\prime}>+<h_{z} h_{z}^{\prime} h_{x} h_{x}^{\prime}>\right)\right) \\
& =\frac{k_{0}^{4}}{(2 \pi)^{2}} z_{0} t_{0} \iint \mathrm{d} r \mathrm{~d} \tau \mathrm{e}^{i \omega \tau-i k_{0} r} \\
& \quad \times\left(\left(k _ { 0 } ^ { 4 } c _ { \mathrm { A } } ^ { 2 } \left(<v_{x}\left(z_{0}, t\right) v_{x}^{\prime}\left(z_{0}+r, t+\tau\right)><h_{z}\left(z_{0}, t\right) h_{z}^{\prime}\left(z_{0}+r, t+\tau\right)>\right.\right.\right. \\
& \left.\quad+<v_{z}\left(z_{0}, t\right) v_{z}^{\prime}\left(z_{0}+r, t+\tau\right)><h_{x}\left(z_{0}, t\right) h_{x}^{\prime}\left(z_{0}+r, t+\tau\right)>\right) \\
& \quad+k_{z}^{2} \omega^{2}\left(<v_{x}\left(z_{0}, t\right) v_{x}^{\prime}\left(z_{0}+r, t+\tau\right)><v_{z}\left(z_{0}, t\right) v_{z}^{\prime}\left(z_{0}+r, t+\tau\right)>\right. \\
& \left.\left.\quad+<h_{z}\left(z_{0}, t\right) h_{z}^{\prime}\left(z_{0}+r, t+\tau\right)><h_{x}\left(z_{0}, t\right) h_{x}^{\prime}\left(z_{0}+r, t+\tau\right)>\right)\right)(15)
\end{aligned}
$$

where we suppose there are no correlations between longitudinal and transverse turbulence, and the fourth order correlation can be reduced to the product of two second order correlations,

$<a_{z} b_{x} a_{z}^{\prime} b_{x}^{\prime}>=<a_{z} a_{z}^{\prime}><b_{x} b_{x}^{\prime}>$ 
Thus we may rewrite

$$
\begin{aligned}
\left|S_{\mathrm{A}}\left(k_{0}, \omega\right)\right|^{2}= & \frac{1}{(2 \pi)^{2}} z_{0} t_{0} \iint \mathrm{d} k^{\prime} \mathrm{d} \omega^{\prime} \\
& \times\left(k_{0}^{2} \omega^{2}\left(u_{\|}^{2} u_{\perp}^{2}+h_{\|}^{2} h_{\perp}^{2}\right)+k_{0}^{4} c_{\mathrm{A}}^{2}\left(u_{\perp}^{2} h_{\|}^{2}+u_{\|}^{2} h_{\perp}^{2}\right)\right) \\
& \times \psi_{\|}\left(k_{0}-k^{\prime}, \omega-\omega^{\prime}\right) \psi_{\perp}\left(k^{\prime}, \omega^{\prime}\right),
\end{aligned}
$$

with

$$
\begin{aligned}
\Psi_{\|} & =\frac{1}{2} E_{\|} \Delta(k, \omega) \\
\Psi_{\perp} & =\frac{1}{2} E_{\perp} \Delta(k, \omega) .
\end{aligned}
$$

Here $u_{\|}$and $h_{\|}$are the parallel fluid turbulent velocity and parallel Alfvén turbulent velocity; $u_{\perp}$ and $h_{\perp}$ are the respective transverse turbulent velocities. All the turbulent velocities are constant here for the elementary tube, but in the whole inhomogeneous tube, it can be adjusted according to the turbulence model. Note that, owing to the deficiency of knowledge on the turbulence feature of convection zone, we prefer to adopt the uniform turbulence model in the whole tube following the previous works. The shortcoming of the application of such a simple turbulence model is compensated by the strong turbulence assumption, where the turbulence strength of magnetofluid should not change greatly depending on the inhomogeneous magnetic field. Moreover, since the acoustic speed and the Alfvén speed are of the same order in our problem, the efficiency of energy exchange between turbulence cells is not determined mainly by the inhomogeneous magnetic field. The modification of the turbulence model is expected in a further work.

Thus, from our knowledge about the turbulence power spectrum, which here satisfies the well-known Kolmogorov spectrum, we can describe the one dimensional longitudinal and transverse turbulence spectra in Eq. (18) as

$$
\begin{aligned}
& E_{\|}=\left\{\begin{array}{ll}
\frac{18}{55 k_{0}}\left(\frac{k}{k_{0}}\right)^{-5 / 3} & \left(k \geq k_{0}\right) \\
\frac{3}{k_{0}}\left(\frac{1}{5}-\frac{k^{2}}{11 k_{0}^{2}}\right) & \left(k<k_{0}\right)
\end{array},\right. \\
& E_{\perp}=\left\{\begin{array}{ll}
\frac{24}{55 k_{0}}\left(\frac{k}{k_{0}}\right)^{-5 / 3} & \left(k \geq k_{0}\right) \\
\frac{3}{k_{0}}\left(\frac{1}{5}+\frac{k^{2}}{11 k_{0}^{2}}\right) & \left(k<k_{0}\right)
\end{array},\right.
\end{aligned}
$$

with $k_{0}=2 \pi / H$, where the turbulent spectra turn into the inertial range, and $H$ is the height of the turbulent cell. The frequency factor $\Delta$ is defined as

$$
\Delta\left(\frac{\omega}{k u_{k}}\right)=\frac{4}{\sqrt{\pi}} \frac{\omega^{2}}{\left|k u_{k}\right|} \mathrm{e}^{-\left(\omega / k u_{k}\right)^{2}} .
$$

\subsection{Monochromatic wave energy flux}

With these expressions about the turbulent source term in Sect. 3.3, we can get the final expression of monochromatic wave energy flux from Eq. (13) as

$$
\begin{gathered}
\frac{\partial^{2}}{\partial \omega \partial z}<F(z, t)>_{t}=\frac{\pi}{32} \frac{\omega^{2}}{c_{\mathrm{A}}^{3}} \rho_{0}\left(u_{\|}^{2} h_{\perp}^{2}+h_{\|}^{2} u_{\perp}^{2}+u_{\|}^{2} u_{\perp}^{2}+h_{\|}^{2} h_{\perp}^{2}\right) \\
\quad \times \iint \mathrm{d} k^{\prime} E_{\|}\left(k_{0}-k^{\prime}, \omega-\omega^{\prime}\right) E_{\perp}\left(k^{\prime}, \omega^{\prime}\right) g\left(k^{\prime},\left|k_{0}-k^{\prime}\right|, \omega\right),
\end{gathered}
$$

where

$$
\begin{aligned}
g\left(k, k^{\prime}, \omega\right)= & \int_{-\infty}^{\infty} \mathrm{d} \omega^{\prime} \Delta\left(k-k^{\prime}, \omega-\omega^{\prime}\right) \Delta\left(k^{\prime}, \omega^{\prime}\right) \\
= & \frac{16}{\pi} \frac{1}{\left|k_{1} u_{1} k_{2} u_{2}\right|^{3}} \int_{-\infty}^{\infty} \mathrm{d} \omega_{1}\left(\omega_{1}^{2}-\omega^{2} / 4\right)^{2} \\
& \times \exp \left(-\frac{\left(\omega_{1}+\omega / 2\right)^{2}}{\left(k_{1} u_{k_{1}}\right)^{2}}-\frac{\left(\omega_{2}-\omega / 2\right)^{2}}{\left(k_{2} u_{k_{2}}\right)^{2}}\right),
\end{aligned}
$$

with $k_{1}=k^{\prime}$ and $k_{2}=k-k^{\prime}$.

Then, we get the wave energy flux spectrum in the local elementary tube. Nevertheless, as mentioned above, not the total wave energy flux generated from the turbulence motion in the convection zone can propagate through the photosphere. If we want to estimate the torsional wave energy available in the heating of chromosphere and corona, we have to take the effect of phase mixing into account, which is the dominant dissipation mechanism of the torsional mode in our problem.

\section{Phase mixing effect in the magnetic flux tube}

Propagation of MHD waves in the magnetofluid may produce some particular instabilities and dissipation owing to the inhomogeneity. Phase mixing is a kind of wave refraction in nature. When the phase velocity of the Alfvén wave depends on the spatial coordinates in the directions perpendicular to equilibrium magnetic field, large gradients of the wave amplitude in the perpendicular directions may be built up. As a consequence, it strongly increases the dissipation of Alfvén wave energy.

The phase mixing effect is a particular property of torsional Alfvén waves, and here we directly cite the result of phase mixing for propagating waves in Heyvaerts \& Priest (1983) with only a slight modification.

The parameters involved are:

Damping Length

$H_{\mathrm{dam}}=\frac{\lambda_{\|}}{2 \pi}\left(6 R_{\mathrm{tot}}\right)^{1 / 3}$,

where, according to the definition of Heyvaerts and Priest,

$R_{\mathrm{tot}}=\frac{\omega a^{2}(x)}{v_{m}+v_{v}}, a(x)=\left(\mathrm{d} \ln k_{\|} / \mathrm{d} x\right)^{-1}$

with viscosity coefficient

$$
\begin{aligned}
v_{v}= & \left(6.858 \times 10^{3}\right)\left(\frac{T}{10^{3} \mathrm{~K}}\right)^{1 / 2} \\
& \times\left(\frac{10^{3}}{B \text { Gauss }}\right)^{2}\left(\frac{\rho}{10^{-6} \mathrm{~g} \mathrm{~cm}^{-3}}\right) \mathrm{m}^{2} \mathrm{~s}^{-1},
\end{aligned}
$$

and magnetic diffusion coefficient

$v_{\mathrm{m}}=\left(3.16 \times 10^{4}\right)\left(\frac{T}{10^{3} \mathrm{~K}}\right)^{-3 / 2} \mathrm{~m}^{2} \mathrm{~s}^{-1}$.

From these formulas above, and assuming the damping length to be the length of the magnetic flux tube, we may deduce the corresponding phase mixing damping frequency,

$\omega_{\mathrm{d}}=\left(\frac{c_{\mathrm{A}}(x)}{H_{\mathrm{dam}}}\right)^{3 / 2}\left(\frac{6 a^{2}(x)}{v_{v}+v_{\mathrm{m}}}\right)^{1 / 2}$. 
Obviously from Eq. (28), only those torsional Alfvén waves with lower frequencies than the phase mixing damping frequency $\omega_{\mathrm{d}}$ can reach the upper solar atmosphere through magnetic flux tube. This part of the wave energy constitutes the net wave energy flux available in the heating of the chromosphere and corona.

Finally, we can integrate Eq. (22) with respect to $\omega$ over the frequency region lower than $\omega_{\mathrm{d}}$ to obtain the net wave energy flux, which is transferred up into chromosphere. In the same way, if we integrate it over the frequency region higher than $\omega_{\mathrm{d}}$, we then get the torsional Alfvén wave energy dissipated in the photosphere. The result of our evaluation is presented in the next section.

\section{Results of some cases}

\subsection{Model parameters of flux tube and convection zone}

To evaluate the quantitative results of energy flux and the phase mixing effect, we give the necessary model parameters first. During the calculation, the integration of Eq. (22) with source depth is simplified to a factor $z$. We choose this value with the same order as the pressure scale height, here $\sim 2 H_{\mathrm{p}}$, and suppose the deeper sources $\left(>2 H_{\mathrm{p}}\right)$ with larger turbulent scale length are negligible in our problem. In our model, the mixing length coefficient is $\alpha^{\prime}=H / H_{\mathrm{p}} \sim 1$ (see Musielak \& Ulmschneider 2001), and the pressure scale height is $H_{\mathrm{p}} \sim$ $150 \mathrm{~km}$. We choose the length of the magnetic flux tube as $500 \mathrm{~km}$, which is the magnitude of the height of the photosphere, to estimate the possible energy carried by torsional waves up to the chromosphere after phase mixing.

The solar observation shows that there exists the horizontal velocity, $\sim 3 \mathrm{~km} \mathrm{~s}^{-1}$ at the surface of photosphere (Muller 1994), and even faster motion interacting with the magnetic flux tubes, which all can be sources to excite MHD waves. The rms values of these velocities are the turbulent velocities in our problem. Huang et al. (1995) suggested a rational range of turbulent velocity as $1 \sim 2 \mathrm{~km} \mathrm{~s}^{-1}$. We choose $\sim 1.5 \mathrm{~km} \mathrm{~s}^{-1}$ in our estimation.

For comparison, we take some parameters similar to those of Musielak. The flux tube temperature $\sim 5770 \mathrm{~K}$, external pressure $p_{\mathrm{e}} \sim 1.17 \times 10^{5} \mathrm{dyn} / \mathrm{cm}^{2}$, and magnetic field at the tube center $B_{0} \sim 1500$ Gauss, all of which are general magnitudes for photospheric magnetic flux tubes. Using them in the formulas in previous sections, such as Eqs. (22) and (28), we can evaluate the results of wave energy flux and dissipation of torsional Alfvén waves.

\subsection{Energy flux spectrum}

Using Eq. (22) and the model parameters given in Sect. 5.1, we can obtain the torsional Alfvén wave energy flux spectrum given in Fig. 2 (where $\zeta=r / R_{0}$ ), which shows that the energy flux density increases with radial coordinate $r$ in the same tube. For example, the maximum of the wave energy flux increases from $4.46 \mathrm{erg} \mathrm{cm}^{-2} \mathrm{~s} \mathrm{~Hz}$ to $2.32 \times 10^{4} \mathrm{erg} \mathrm{cm}^{-2} \mathrm{~s} \mathrm{~Hz}$, while the respective $r$ moves from the tube center to $400 \mathrm{~km}(\zeta=0.8)$.

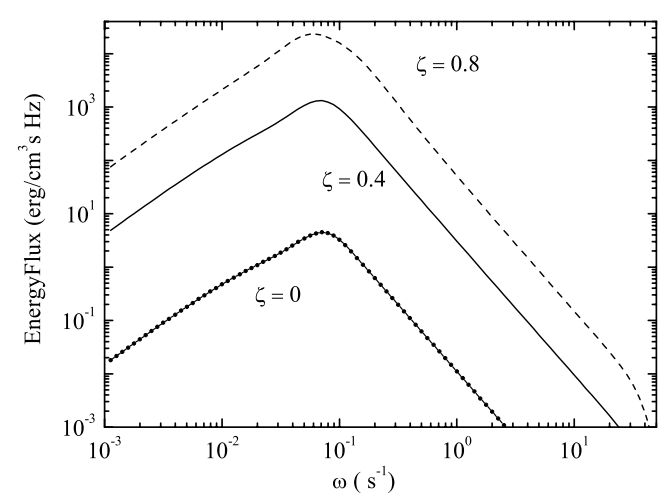

Fig. 2. Wave energy flux spectra in different $r$, denoted by $\zeta=r / R_{0}$ with the magnetic radial decline coefficient $\alpha=2$ and radius $R_{0}=$ $500 \mathrm{~km}$. The wave energy flux is the total torsional Alfvén wave energy flux generated from turbulent source.

Another aspect is that the profile of the spectrum tends to move towards the lower frequency direction when $r$ increases. From Fig. 2, when $\zeta$ increases from 0 to 0.8 , the corresponding frequency, where the energy flux approaches its maximum, changes slightly from $\omega_{\max }=7.08 \times 10^{-2} \mathrm{~Hz}$ to $5.62 \times 10^{-2} \mathrm{~Hz}$. However, the respective phase mixing damping frequency $\omega_{\mathrm{d}}$, given by Eq. (28), decreases rapidly. When $\zeta$ is $0,0.4$, and 0.8 , the $\omega_{\mathrm{d}}$ is $41.32,3.64$, and 0.43 , respectively. Therefore, the strength of phase mixing at the exterior is much stronger than that at the interior of the flux tube.

\subsection{The net energy flux and comparison to previous work}

The problem of how the fluid motion energy, carried by MHD waves from the convection zone, propagates through magnetic flux tubes up into the upper solar atmosphere has attracted solar physicists for many years. Musielak et al. (1989) investigated the interaction between thin magnetic flux tubes and the ambient turbulent medium, and worked out the longitudinal wave energy flux of about the order of $\sim 10^{7} \mathrm{erg} \mathrm{cm}^{-2} \mathrm{~s}^{-1}$. Applying the Lighthill-Stein theory in the sunspot model, Lee (1993) evaluated the longitudinal and Alfvén wave energy flux to be about $\sim 10^{8} \mathrm{erg} \mathrm{cm}^{-2} \mathrm{~s}^{-1}$. A larger energy flux given in Muller (1994) is about $\sim 10^{10} \mathrm{erg} \mathrm{cm}^{-2} \mathrm{~s}^{-1}$, but the difference may come from the different initial conditions. Based on the Spruit (1981) magnetic flux tube equations, Musielak \& Ulmschneider (2001) estimated the transverse modes energy flux to be about the order of $\sim 10^{8} \mathrm{erg} \mathrm{cm}^{-2} \mathrm{~s}^{-1}$. Progress was also made by numerical simulations, such as ny Huang et al. (1995), which simulated the nonlinear wave excitation response to the transverse perturbation at different heights relative to the magnetic flux tubes in the solar atmosphere, and obtained an energy flux density to be about the order of $\sim 10^{9} \mathrm{erg} \mathrm{cm}^{-2} \mathrm{~s}^{-1}$.

Unlike these works mentioned above, our emphasis here is on the torsional modes generated from an inhomogeneous magnetic flux tube, and for a tube $R_{0}=500 \mathrm{~km}$, we gain the average net energy flux density $\widehat{E}$ :

1. when $\alpha=1, \widehat{E} \sim 2.31 \times 10^{7} \mathrm{erg} \mathrm{cm}^{-2} \mathrm{~s}^{-1}$; 


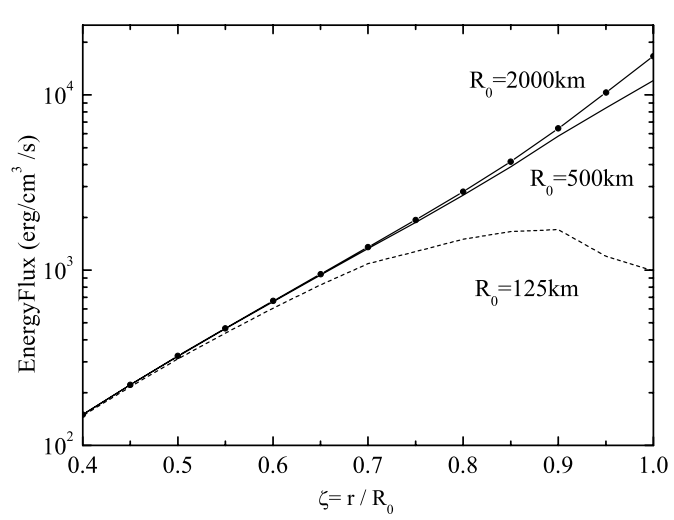

Fig. 3. Radial distribution of wave energy flux density in flux tubes with different radii, when $\alpha=2$. For $R_{0}=125 \mathrm{~km}$, the energy flux density decreases near the edge of tube, due to the stronger enhancement of the phase mixing effect, which is far beyond the increase wave energy excitation.

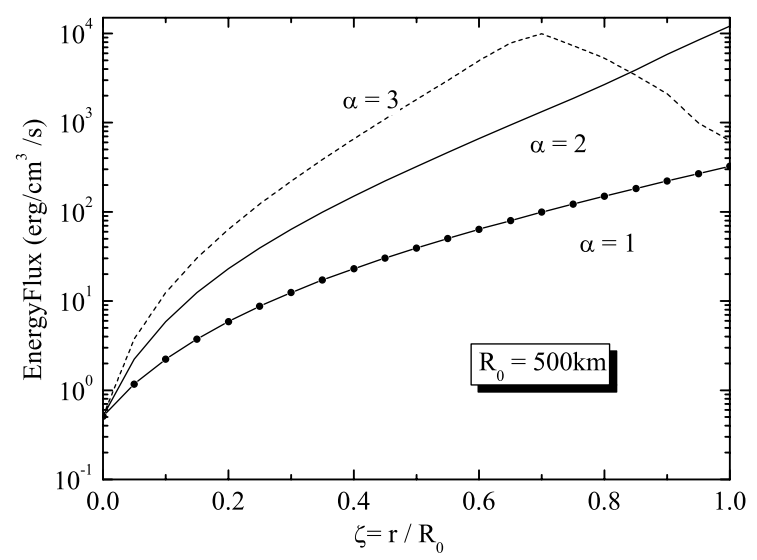

Fig. 4. Radial distribution of wave energy flux density with different $\alpha$, when radius $R_{0}=500 \mathrm{~km}$. For the stronger inhomogeneous magnetic field where $\alpha=3$, the energy flux density fades greatly owing to the stronger effects of phase mixing.

2. when $\alpha=2, \widehat{E} \sim 4.96 \times 10^{8} \mathrm{erg} \mathrm{cm}^{-2} \mathrm{~s}^{-1}$;

3. when $\alpha=3, \widehat{E} \sim 7.41 \times 10^{8} \mathrm{erg} \mathrm{cm}^{-2} \mathrm{~s}^{-1}$,

where we estimate three tube models with different $\alpha$, the radial decline coefficient of the magnetic field, which is partially due to the uncertainty of the inhomogeneity. The results above show that the torsional modes carry enough energy up to the chromosphere, so they play an important role in the processes of energy transfer from the convection zone to the upper solar atmosphere, and is a likely cause of the heating of the chromosphere and corona.

Figures 3 and 4 show that the exterior region of the tube, where $r / R_{0}>0.5$, contributes largely to the total wave energy flux. The wave energy flux density increases rapidly with increasing coordinate $r$, which is due to the relaxation of strong magnetic field confinement in the tube center. It is similar to the stiffness effect on the flux tube wave excitation (see Musielak \& Ulmschneider 2001).

As mentioned above, we neglect the stratification effect of the solar atmosphere, which will add a cutoff frequency into the wave equations (see Lee 1993), $\omega_{\text {cutoff }}=c_{\mathrm{A}} / 2 H_{\mathrm{p}} \sim 10^{-2}$.

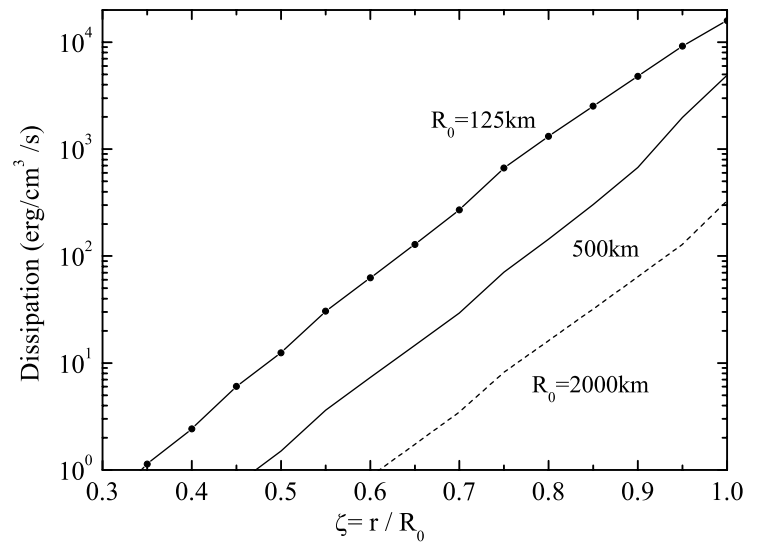

Fig. 5. Radial distribution of wave energy dissipation from phase mixing to different radii, $R_{0}=125 \mathrm{~km}, 500 \mathrm{~km}$, and $2000 \mathrm{~km}$, when $\alpha=2$.

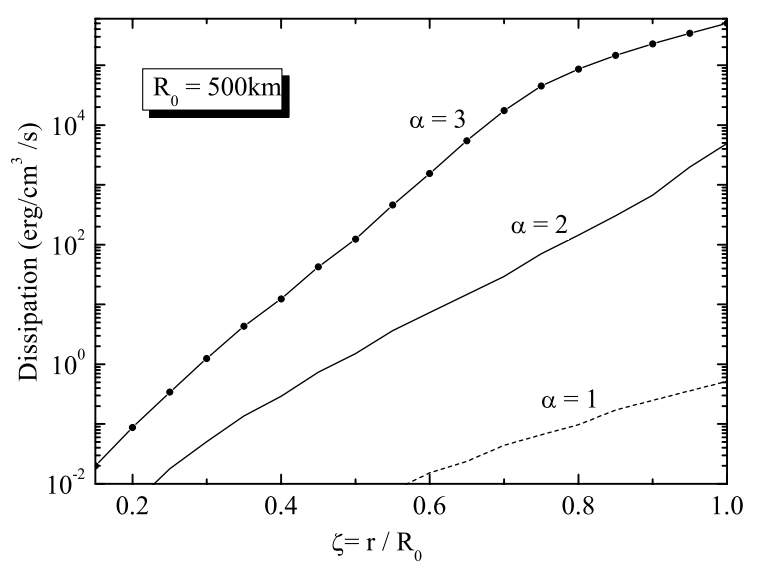

Fig. 6. Radial distribution of wave energy dissipation from phase mixing to different magnetic radial decline coefficient $\alpha$, when radius $R_{0}=500 \mathrm{~km}$.

The MHD waves with frequencies lower than $\omega_{\text {cutoff }}$ can never transfer energy up to the chromosphere. Thus, the cutoff frequency may block the transfer of a part of the energy, which should be included in further research. Moreover, we note that the energy fluxes given here are the net wave energy fluxes, and those parts of energy dissipated by phase mixing are given below.

\subsection{Dissipation of phase mixing}

As a special property of torsional Alfvén waves, phase mixing should be taken into account when describing their propagation through an inhomogeneous magnetic flux tube. Here, we evaluate only the simple phase mixing effect, and obtain its heating efficiency for the flux tube.

For the flux tube with a magnetic radial decline coefficient $\alpha=2$, the average phase mixing dissipation $\widehat{D}_{\mathrm{p}}$ is:

1. when $R_{0}=125 \mathrm{~km}, \widehat{D}_{\mathrm{p}}=9.44 \times 10^{7} \mathrm{erg} \mathrm{cm}^{-2} \mathrm{~s}^{-1}$;

2. when $R_{0}=500 \mathrm{~km}, \widehat{D}_{\mathrm{p}}=1.64 \times 10^{7} \mathrm{erg} \mathrm{cm}^{-2} \mathrm{~s}^{-1}$;

3. when $R_{0}=2000 \mathrm{~km}, \widehat{D}_{\mathrm{p}}=1.49 \times 10^{6} \mathrm{erg} \mathrm{cm}^{-2} \mathrm{~s}^{-1}$.

Consistent with exterior enhancement of phase mixing as described in Sect. 6.2, Figs. 5 and 6 show also the similar 
edge-enhancing effect of phase mixing (see Roberts 1992). However, no corresponding bright rings were observed around thin flux tubes or sunspots, which can be explained with the deformation of the tube edge owing to the interaction with the surrounding medium, so that simple phase mixing fails for the more complicated coupling modes (Parker 1991). In such cases, the wave dissipation can be explored through other kinds of functions, e.g., resonance absorption (Lee \& Roberts 1986), but they are beyond our study here.

From the results given above, we find strong dependence of phase mixing on $\alpha$, which show the inhomogeneity of the tube. Comparing Fig. 4 with Fig. 6, when $\alpha=1$, phase mixing can be ignored; but when $\alpha=3$, for a tube with $R_{0}=500 \mathrm{~km}$, the energy dissipation of phase mixing is overwhelming, and drags the net wave energy flux down fast when $\zeta>0.7$.

According to our results, thin flux tubes (here, $R_{0}=$ $125 \mathrm{~km}$ ) are affected more strongly by phase mixing, which can even deplete the wave energy of torsional modes. In the tubes with the same magnetic radial decline coefficient $\alpha=2$, e. g., for a flux tube with $R_{0}=2000 \mathrm{~km}$, the dissipation is only about $1 \%$ of the total wave energy generated from a turbulent source, while this value is about $14 \%$ for a tube with $R_{0}=500 \mathrm{~km}$, and greater than $70 \%$ for a tube with $R_{0}=125 \mathrm{~km}$. But just like the consideration before, thin magnetic flux tubes are more prone to be distorted under external disturbance, so that its torsional modes couple with other modes, and the strong heating effect perhaps deviates the magnetofluid parameters greatly from their initial value in our models. Therefore, more careful consideration is necessary for this problem.

\section{Conclusion and discussion}

In summary, the results and main points of our work are listed as follows:

1. In this paper, we apply the Lighthill-Stein theory to the inhomogeneous magnetic flux tube, and find a significant role for the torsional mode in the energy transfer from convection zone to chromosphere.

2. In weak inhomogeneous magnetic flux tubes, where $\alpha=$ 1 , the torsional wave energy flux is of the order of $10^{6} \mathrm{erg} \mathrm{cm}^{-2} \mathrm{~s}^{-1}$, which can meet the energy necessary to the quiet chromospheric and coronal heating.

3. As for moderate inhomogeneous tubes, where $\alpha=2$, the energy flux of torsional modes increases to the order of $10^{8} \mathrm{erg} \mathrm{cm}^{-2} \mathrm{~s}^{-1}$, which is enough to supply the heating of active regions.

4. The phase mixing dissipation in strong inhomogeneous tubes $\alpha=3$ can be a possible mechanism to heat the solar atmosphere, which can use most of the wave energy in the tube.

5. We find that the energy flux increases with the coordinate $r$, which is codetermined by the strength of wave generation and inhomogeneous dissipation. This result is instructive when studying the footpoint motion of the coronal loop.

We explore local turbulence excitation in the magnetic flux tube during torsional Alfvén wave generation. But the excitation of other modes, such as longitudinal and transverse modes, make the relevant formulae of inhomogeneous plasma more perplexing, and the interaction between the magnetic flux tube and the surrounding medium is another promising energy source. When we consider those more reliable wave modes $k_{\mathrm{r}} \neq 0$, the coupling with fast waves should be taken into account (De Groof \& Goossens 2002). Furthermore, the additional instabilities and the nonlinear effect (Ulmschneider \& Musielak 1998; Lundberg 1994), arising from the inhomogeneity, will complicate the process of wave propagation, too, and therefore, many more studies are necessary to understand the problem of nonuniform hydromagnetic parameters.

Acknowledgements. This work was supported by the National Natural Science Foundation of China under grants 49390450 \& 499925412 , and Chinese National Basic Research Project (Grant No. NkBRSF G20000984).

\section{References}

Berghmans, D., \& De Bruyne, P. 1995, ApJ, 453, 495

Berghmans, D., \& Tirry, W. J. 1997, A\&A, 325, 318

Collins, W. 1989a, ApJ, 337, 548

Collins, W. 1989b, ApJ, 343, 499

Collins, W. 1992, ApJ, 384, 319

De Groof, A., Paes, K., \& Goossens, M. 2002, A\&A, 386, 681

De Groof, A., \& Goossens, M. 2002, A\&A, 386, 691

Heyvaerts, J., \& Priest, E. R. 1983, A\&A, 117, 220

Hollweg, J. V. 1991, in Mechanics of Chromospheric and Coronal Heating, ed. P. Ulmschneider, E. R. Priest, \& R. Rosner (SpringerVerlag, Heidelberg), 423

Huang, P., Musielak, Z. E., \& Ulmschneider, P. 1995, A\&A, 279, 579

Lee, J. W. 1993, ApJ, 404, 372

Lee, M. A., \& Roberts, B. 1986, ApJ, 301, 430

Lighthill, M. J. 1952, Proc. Roy. Soc. London A, 211, 564

Lundberg, J. 1994, Sol. phys., 154, 215

Muller, R., Roudier, Th., Vigneau, J., \& Auffret, H. 1994, A\&A, 283, 232

Musielak, Z. E., Rosner, R., \& Ulmschneider, P. 1989, ApJ, 241, 625

Musielak, Z. E., Rosner, R., Gail, H. P., \& Ulmschneider, P. 1995, ApJ, 448,865

Musielak, Z. E., Rosner, R., \& Ulmschneider, P. 2000, ApJ, 541, 410

Musielak, Z. E., \& Ulmschneider, P. 2001, A\&A, 370, 541

Nakariakov, V. M., Roberts, B., \& Murawski, K. 1997, Sol. Phys., 175, 93

Narain, U., \& Ulmschneider, P. 1990, Space Sci. Rev., 54, 377

Narain, U., \& Ulmschneider, P. 1996, Space Sci. Rev., 75, 453

Parker, E. N. 1991, ApJ, 376, 355

Roberts, B., \& Ulmschneider, P. 1997, in Solar and Heliospheric Plasma Physics, ed. G. M. Simett, C. E. Alissandrakis, \& L. Vlahos (Springer Verlag, Berlin), 75

Roberts, B. 1992, in Sunspots: Theory and Observation, ed. J. H. Thomas, \& N. O. Weiss (Kluwer, Dordrecht), NATO ASI Series, 303

Ruderman, M. S., Goldstein, M. L., Roberts, D. A., Deane, A., \& Ofman, L. 1999, J. Geophys. Res., 104, 17057

Sakai, J. I., Mizuhata, Y., Kawata, T., \& Cramer, N. F. 2000, ApJ, 544, 1108

Spruit, H. C. 1981, A\&A, 98, 155

Spruit, H. C. 1982, Sol. Phys., 75, 3

Stein, R. F. 1967, Sol. Phys., 2, 385

Ulmschneider, P., \& Musielak, Z. E. 1998, A\&A, 338, 311 